\title{
Importance of rate control or rate regulation for improving exercise capacity and quality of life in patients with permanent atrial fibrillation and normal left ventricular function: a randomised controlled study
}

\author{
T Levy, S Walker, M Mason, P Spurrell, S Rex, S Brant, V Paul
}

\begin{abstract}
Objective-To determine the importance of rhythm regulation or rate control in patients with permanent atrial fibrillation (AF) and normal left ventricular function.

Patients and interventions-Thirty six patients with a mixed fast and slow ventricular response rate to their AF were randomised to either His bundle ablation (HBA) and VVIR pacemaker (HBA group) or VVI pacemaker and atrioventricular modifying drugs (Med group). Outcomes assessed at one, three, six, and 12 months included exercise duration and quality of life.

Results-Exercise duration significantly improved from baseline in both groups. There was no difference in outcome between the groups (Med $+40 \% v$ HBA $+20 \%, p=N S$ ). The heart rate profile on exercise was similarly slowed in both groups compared to baseline. Quality of life significantly improved in both treatment arms for the modified Karolinska questionnaire (KQ) $(\mathrm{Med}+50 \% v \mathrm{HBA}+50 \%, \mathrm{p}=\mathrm{NS})$ and the Nottingham health profile (NHP) (Med $+40 \% v$ HBA $+20 \%$, p = NS). However, for the individual symptom scores of each questionnaire more were improved in the Med group (KQ-Med 6 improved $v$ HBA 4, NHP-Med $3 v$ HBA 1). Left ventricular function was equally preserved by both treatments during follow up.

Conclusion-In these patients control of ventricular response rate with either HBA + VVIR pacemaker or atrioventricular modifying drugs + VVI pacemaker will lead to a significant improvement in exercise duration and quality of life. Rhythm regulation by HBA did not confer additional benefit, suggesting rate control alone is necessary for the successful symptomatic treatment of these patients in permanent AF.
\end{abstract}

(Heart 2001;85:171-178)

Keywords: ablation; atrial fibrillation; pacemaker; atrioventricular modifying drugs

Radiofrequency catheter ablation of the His bundle (or atrioventricular node) and implantation of a rate responsive pacemaker (VVIR pacemaker) has been shown to improve symptoms, quality of life, and exercise duration in patients with permanent atrial fibrillation (AF) and medically refractory fast ventricular response. ${ }^{1-3}$ Left ventricular function if impaired can also improve following ablation. ${ }^{24-10}$ This benefit of His bundle ablation (HBA) may be caused by improved ventricular rate control, ventricular rhythm regulation or a combination of both.

There is increasing evidence suggesting a detrimental effect from an irregular ventricular rhythm. Studies comparing irregular right ventricle pacing to a regular paced rhythm with the same mean heart rate have demonstrated a decrease in cardiac output and an increase in wedge pressure during the irregular pacing. ${ }^{112}$ Furthermore prospective studies of HBA with VVIR pacemaker implantation in patients with permanent $\mathrm{AF}$ and impaired left ventricular function with controlled ventricular rates (60100 beats/min (bpm)) at baseline have shown an improved quality of life and ejection fraction following ablation, supporting the importance of ventricular rate regulation. ${ }^{13}{ }^{14}$

However, there are disadvantages to atrioventricular nodal ablation including that it is permanent and irreversible. The patient therefore becomes pacemaker dependent, with the associated risks of pacemaker failure, lead malfunction, and need for re-implantation. Ventricular pacing also leads to asynergic ventricular contraction which can be negatively inotropic, cause mitral regurgitation and, in the long term, lead to the development of myocardial perfusion defects associated with abnormal wall motion and impaired left ventricular function. ${ }^{15-18}$

Many patients in permanent $\mathrm{AF}$ have a mixed ventricular response rate with both symptomatic fast and slow episodes. These patients are not drug refractory but rather drug intolerant, as additional atrioventricular modifying drugs exacerbate their bradycardia. Treatment options include implantation of a permanent pacemaker (VVI) to prevent the bradycardia with the addition of atrioventricular modifying drugs to control the fast ventricular rates. An alternative strategy is HBA and VVIR pacemaker implantation. This has the potential advantage of achieving optimal ventricular rate control and rhythm regulation without the need for potentially negatively inotropic atrioventricular depressant drugs.

In this randomised study we compared these two treatment options in this type of patient with permanent AF. In addition these patients 
also had a normal left ventricular function. Our aim was to determine if exercise capacity and quality of life could be improved by ventricular rate control alone (drugs plus VVI pacing) or whether the addition of rhythm regulation (ablation) added any further benefit.

\section{Methods}

PATIENTS

Between June 1996 to April 199836 patients (mean (SD) age 69 (7) years, 14 female) were referred to our institution for implantation of a permanent pacemaker after assessment by their local cardiologist. All patients were in permanent $\mathrm{AF}$ ( $>6$ months). They all had a symptomatic fast ventricular response rate (rest rate $>100 \mathrm{bpm}$ or rate $>80 \%$ target heart rate after two minutes on exercise testing) to their $\mathrm{AF}$ that could not be controlled by drugs as they induced symptomatic bradycardic episodes (rate $<40 \mathrm{bpm}$ or pause $>3$ seconds). Baseline 24 hour tape confirmed these symptomatic episodes in the presence of their baseline medication.

Further inclusion criteria included consenting patients (age $>18$ years) that were fully ambulant with mobility not limited by other medical conditions such as arthritis, lung disease or previous stroke. Exclusion criteria included limited life expectancy, history of sustained ventricular arrhythmias, symptomatic angina, pregnancy, recent myocardial infarction or major surgery within six months.

STUDY PROTOCOL

All patients had a VVIR pacemaker inserted. They were then randomised to one of two groups:

Medical treatment (Med group)-The pacemaker was programmed to VVI base rate $70 \mathrm{bpm}$ with the addition of further atrioventricular modifying medication before discharge to improve ventricular rate control. First choice drugs were verapamil or diltiazem to maximum tolerated doses, with the addition of digoxin if rate control remained problematic. If these were ineffective or poorly tolerated $\beta$ blockers could be substituted or added. Changes in medications were allowed during the study in response to clinical symptoms or poor rate control seen at follow up visits.

Ablation (HBA group) - These patients underwent successful His bundle ablation from the venous side in all cases at the time of pacemaker implantation. The pacemaker was programmed to VVIR base rate $60 \mathrm{bpm}$, upper rate $85 \%$ of age predicted (220 minus age). All atrioventricular modifying drugs were discontinued in these patients before discharge unless they were used for other clinical indications such as heart failure, hypertension or angina.

Patients were randomised in groups of six, by selecting a card blindly from a container with initially six cards present (three medical, three ablation). The study was not blinded. Our hypothesis was that the HBA group would achieve a greater improvement in exercise duration and quality of life compared to the Med group, due to an equivalent effect on rate
Table 1 Baseline features

\begin{tabular}{|c|c|c|}
\hline & $\begin{array}{l}\text { Med } \\
(n=18)\end{array}$ & $\begin{array}{l}H B A \\
(n=18)\end{array}$ \\
\hline Age (years) & $69(7)$ & $68(8)$ \\
\hline Sex (male/female) & $11 / 7$ & $11 / 7$ \\
\hline Duration AF (years) & $3.5(3.8)$ & $3.8(4.0)$ \\
\hline \multicolumn{3}{|l|}{ Aetiology AF } \\
\hline Mitral valve disease & 5 & 7 \\
\hline Ischaemia & 5 & 5 \\
\hline Hypertension & 1 & 1 \\
\hline Idiopathic & 7 & 5 \\
\hline \multicolumn{3}{|l|}{ Medication } \\
\hline ACE inhibitors & 7 & 7 \\
\hline Diuretics & 10 & 8 \\
\hline Warfarin & 11 & 14 \\
\hline Aspirin & 4 & 2 \\
\hline \multicolumn{3}{|l|}{ Nodal modifying drugs } \\
\hline Digoxin & 14 & 13 \\
\hline$\beta$ Blockers & 2 & 1 \\
\hline Verapamil & 2 & 2 \\
\hline Diltiazem & 1 & 1 \\
\hline Nil & 4 & 4 \\
\hline \multicolumn{3}{|l|}{ Echocardiogram } \\
\hline Systolic diameter (mm) & $34(6)$ & $37(7)$ \\
\hline Diastolic diameter (mm) & $49(7)$ & $51(8)$ \\
\hline Ejection fraction $(\%)$ & $67(9)$ & $61(9)$ \\
\hline Left atrial size $(\mathrm{mm})$ & $47(7)$ & $47(8)$ \\
\hline
\end{tabular}

This shows both groups are matched. Results shown are mean (SD).

ACE, angiotensin converting enzyme.

control but with the addition of ventricular rhythm regulation. The local ethical committee approved the study.

Table 1 gives details of the baseline characteristics in both randomised groups, which were matched. Overall at baseline no atrioventricular modifying drugs were used in eight patients $(22 \%$, Med $n=4$, HBA $n=4)$, one drug was used in 20 patients $(56 \%$, Med $\mathrm{n}=10$, HBA $\mathrm{n}=10$ ), whereas eight patients required two drugs $(22 \%$, Med $n=4$, HBA $\mathrm{n}=4)$. Digoxin was used in $75 \%$ of patients (Med $n=14$, HBA $n=13$ ), either singularly $(n=19)$ or in combination $(n=8)$. Baseline left ventricular function was assessed with echocardiography within the first 24 hours after pacemaker implantation, with the pacemaker programmed to VVI base rate $100 \mathrm{bpm}$ to allow standardisation. The ejection fraction was within normal limits for both groups; no patient had an ejection fraction $<45 \%$ and only four patients (Med $\mathrm{n}=2$, HBA $\mathrm{n}=2$ ) had an ejection fraction $<50 \%$.

Assessments were performed at baseline, one, three, six, and 12 months. These included cardiopulmonary exercise testing, quality of life questionnaires (a modified Karolinska questionnaire (KQ), a cardiac specific and the Nottingham health profile (NHP)), a general health questionnaire, 24 hour tape, and echocardiography. The baseline assessment, other than the echocardiogram, was performed before pacemaker implantation.

The main outcomes of the study were intrapatient (intra-group) comparison of follow up results with patients' baseline measurements to detect significant changes. Inter-group (Med $v$ HBA) comparisons were also made at baseline and all follow up visits.

CARDIOPULMONARY EXERCISE TESTING

Patients performed a symptom limited treadmill exercise test using the CAEP (chronotropic assessment exercise) protocol with 
continuous electrocardiographic monitoring. Measurements recorded included: resting heart rate, heart rate at one minute intervals for first three minutes, peak heart rate, percentage of predicted heart rate reached, exercise duration (in minutes), and $\mathrm{VO}_{2} \mathrm{max}(\mathrm{ml} / \mathrm{min} /$ $\mathrm{kg}$ ) (defined as the maximum oxygen uptake at any stage during exercise). Oxygen consumption $\left(\mathrm{VO}_{2}, \mathrm{ml} / \mathrm{kg} / \mathrm{min}\right)$ was continuously measured and analysed using an automated breath by breath system (Medgraphics Cardiopulmonary Exercise System CPX/D Series 2; Medgraphics, Kings Lynn, UK).

MODIFIED KAROLINSKA QUESTIONNAIRE (KQ)

$\mathrm{KQ}$ is a cardiac specific questionnaire that has been validated for pacemaker patients. ${ }^{19} \mathrm{~Pa}$ tients were asked to grade their response between 0 (no symptoms) and 10 (severe symptoms) to a series of questions on: chest pain (total score $0-40$ ), palpitations $(0-30)$, dizziness (0-20), shortness of breath (0-30), general health (0-10), and activity level (0-10). This gives a total score between 0 (asymptomatic) and 140 (very symptomatic).

NOTTINGHAM HEALTH PROFILE (NHP)

NHP is a general quality of life questionnaire validated for cardiac patients. ${ }^{2021}$ It is divided into two parts. The first consists of 38 statements describing levels of physical, social or emotional distress that are grouped into six dimensions: physical mobility, pain, sleep, energy, social isolation, and emotional reaction. Patients answer yes or no to each question. A score (0-100) is then calculated for each dimension from these answers, and the higher the score the greater the limitation. The six individual scores from each dimension can be added together to give a total NHP score (0-600) - a higher score means greater limitation.

The second part lists seven aspects of life that may be effected by a patient's health: occupation, ability to perform jobs around the home, social life, home relationships, sex life, hobbies/ interest, and holidays. Patients answer yes or no to whether their health interferes with these activities.

24 HOUR TAPE

Patients were fitted with a Siemens 24 hour ECG recorder. Analysis was performed on a Pathfinder 700 (Reynolds Medical, Hertford, UK) tape analyser to determine mean heart rate over 24 hours and maximum and minimum heart rates at all follow ups. Baseline 24 hour tape was not analysed in this way.

ECHOCARDIOGRAM

All patients had their pacemaker reprogrammed to VVI base rate $100 \mathrm{bpm}$ for the echocardiogram to give a standardised heart rate at all studies for all patients. Subjects were examined by $M$ mode, two dimensional (cross sectional), and pulsed wave Doppler $(2.5 \mathrm{MHz}$ transducer) on a Hewlett-Packard (model Sonos 1500) ultrasound machine with simultaneous ECG monitoring. Measurements in- cluded diastolic diameter, systolic diameter, ejection fraction, and left atrial diameter.

STATISTICAL ANALYSIS

Values are given as mean (SD). Comparison between follow up and baseline results (intragroup) was with the Wilcoxon test for continuous variables (a non-parametric test for two related samples) and the Fishers exact probability test (two tail) for non-continuous variables. Comparison between each group (inter-group, Med $v$ HBA) is by the use of the Mann-Whitney test for continuous variables (a non-parametric test for two independent samples) and the Fishers exact probability test (two tail) for non-continuous variables.

\section{Results}

In the Med group follow up was complete at one and three months $(n=18)$ and available for 16 patients at six and 12 months. One patient developed breast cancer after five months preventing further follow up, and another refused further follow up after a three month check. In the HBA group follow up was complete at one, three, and six months $(\mathrm{n}=18)$ and available in 16 patients at 12 months. One patient died at nine months and another refused further follow up after a six month check.

In the Med group rate control was achieved by single drug treatment in five patients $(28 \%$, verapamil $\mathrm{n}=1$, diltiazem $\mathrm{n}=2, \beta$ blockers $\mathrm{n}=1$, and digoxin $\mathrm{n}=1$ ), two drug treatment in 11 patients $(61 \%$, verapamil + digoxin $\mathrm{n}=7$, diltiazem + digoxin $\mathrm{n}=3, \beta$ blockers + digoxin $n=1)$, and three drug treatment in two patients $(11 \%$, verapamil + digoxin $+\beta$ blockers $n=1$, diltiazem + digoxin $+\beta$ blockers $n=1)$. All patients had an increase in either the dosage or number of drugs used following pacemaker implantation. No patient from the medical group underwent ablation during the study period.

In the HBA group all atrioventricular modifying drugs were discontinued.

\section{CARDIOPULMONARY EXERCISE TESTING}

In the Med group there was a significant improvement in exercise duration at one month that was maintained throughout the study (table 2, fig 1). Overall exercise duration increased by approximately $40 \%$. There was no significant improvement in $\mathrm{Vo}_{2}$ max. The peak heart rate on exercise or percentage maximum predicted did not significantly change from baseline at any stage. However, there was a significant change in the heart rate profile on exercise (fig 2). Although resting rate did not change compared to baseline, the rate at one minute intervals over the first three minutes was significantly slower at all follow up visits.

In the HBA group there was again a significant improvement in exercise duration at one month that was maintained throughout the study (table 2, fig 1). Overall exercise duration increased by approximately $20 \%$. There was no significant change in $\mathrm{VO}_{2} \max$. There was a significant decrease in the peak heart rate obtained on exercise and the percentage maxi- 
Table 2 Results for treadmill exercise testing for both groups (Med, medical group; HBA, ablation group) at baseline line and all follow ups

\begin{tabular}{|c|c|c|c|c|c|c|c|c|c|c|}
\hline & \multicolumn{2}{|l|}{ Baseline } & \multicolumn{2}{|l|}{1 month } & \multicolumn{2}{|l|}{3 months } & \multicolumn{2}{|l|}{6 months } & \multicolumn{2}{|l|}{12 months } \\
\hline & $\begin{array}{l}\text { Med } \\
(n=18)\end{array}$ & $\begin{array}{l}H B A \\
(n=18)\end{array}$ & $\begin{array}{l}\text { Med } \\
(n=18)\end{array}$ & $\begin{array}{l}H B A \\
(n=18)\end{array}$ & $\begin{array}{l}\text { Med } \\
(n=18)\end{array}$ & $\begin{array}{l}H B A \\
(n=18)\end{array}$ & $\begin{array}{l}\text { Med } \\
(n=16)\end{array}$ & $\begin{array}{l}H B A \\
(n=18)\end{array}$ & $\begin{array}{l}\text { Med } \\
(n=16)\end{array}$ & $\begin{array}{l}H B A \\
(n=16)\end{array}$ \\
\hline Exercise duration (mins) & $4.9(2.9)$ & $5.3(2.8)$ & $6.9(2.4)^{\star}$ & $6.2(2.6)^{\star}$ & $7.0(2.1)^{\star}$ & $6.6(2.7)^{\star}$ & $7.0(2.8)^{\star}$ & $6.6(3.0)^{\star}$ & $7.1(2.6)^{\star}$ & $6.8(2.8)^{\star}$ \\
\hline $\mathrm{VO}_{2} \max (\mathrm{ml} / \mathrm{min} / \mathrm{kg})$ & $17(4)$ & $16(3)$ & $19(4)$ & $16(3)$ & $19(4)$ & $16(3)$ & $18(4)$ & $16(3)$ & $18(4)$ & $16(3)$ \\
\hline Peak heart rate (bpm) & $148(32)$ & $160(26)$ & $139(22)$ & $114(20)^{\star}$ & $133(22)$ & $122(22)^{\star}$ & $140(22)$ & $120(23)^{\star}$ & $132(29)$ & $120(20)^{\star}$ \\
\hline$\%$ predicted peak heart rate & $100(21)$ & $110(22)$ & $93(16)$ & $77(14)^{\star}$ & $89(13)$ & $84(18)^{\star}$ & $94(15)$ & $81(14)^{\star}$ & $88(18)$ & $80(13)^{\star}$ \\
\hline Heart rate rest (bpm) & $85(13)$ & $90(26)$ & $78(14) \dagger$ & $67(10)^{\star} \dagger$ & $77(11) \dagger$ & $67(10)^{\star} \dagger$ & $78(14) \dagger$ & $67(11)^{\star} \dagger$ & $76(10) \dagger$ & $66(10)^{\star} \dagger$ \\
\hline Heart rate 1 minute (bpm) & $113(21)$ & $118(28)$ & $95(20)^{\star}$ & $81(15)^{\star}$ & $88(16)^{\star}$ & $87(24)^{\star}$ & $90(19)^{\star}$ & $84(20)^{\star}$ & $87(17)^{\star}$ & $80(17)^{\star}$ \\
\hline Heart rate 2 minutes (bpm) & $123(21)$ & $130(25)$ & $100(22)^{\star}$ & $86(20)^{\star}$ & $96(15)^{\star}$ & $84(21)^{\star}$ & $94(20)^{\star}$ & $87(23)^{\star}$ & $90(20)^{\star}$ & $83(19)^{\star}$ \\
\hline Heart rate 3 minutes (bpm) & $130(26)$ & $135(23)$ & $105(18)^{\star}$ & $86(21)^{\star}$ & $103(20)^{\star}$ & $97(16)^{\star}$ & $100(21)^{\star}$ & $93(21)^{\star}$ & $100(17)^{\star}$ & $89(20)^{\star}$ \\
\hline
\end{tabular}

${ }^{\star} \mathrm{p}<0.05$ for intra-group comparison with baseline measurements; $t \mathrm{p}<0.05$ for inter-group comparison of Med $v$ HBA.

mum predicted at all follow ups. There was again a significant change in the heart rate profile on exercise (fig 2). Compared to baseline both resting heart rate and rates at one minute intervals over the first three minutes were all significantly slower at all follow ups.

For inter-group comparison (Med $v$ HBA) there was no significant differences in any baseline results or between the groups in follow up (full statistical results not shown) except for the resting heart rate before exercise. This was

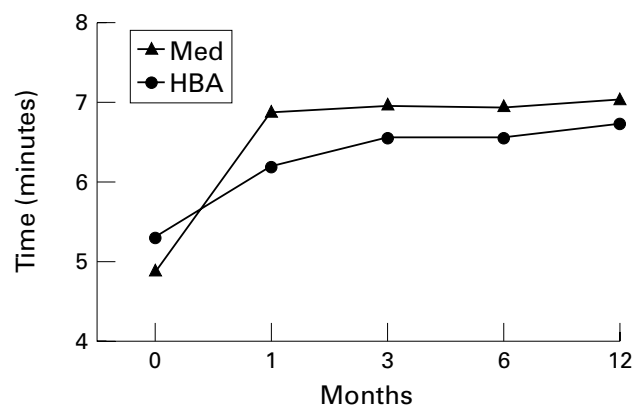

Figure 1 Exercise duration in seconds on treadmill testing for both groups (Med, medical; HBA, ablation) at baseline and at all follow ups. Intra-group comparison for Med and HBA group showed a significant improvement compared to baseline at all follow ups. Inter-group comparison (Med $v$ $H B A$ ) showed no significant difference at any time.

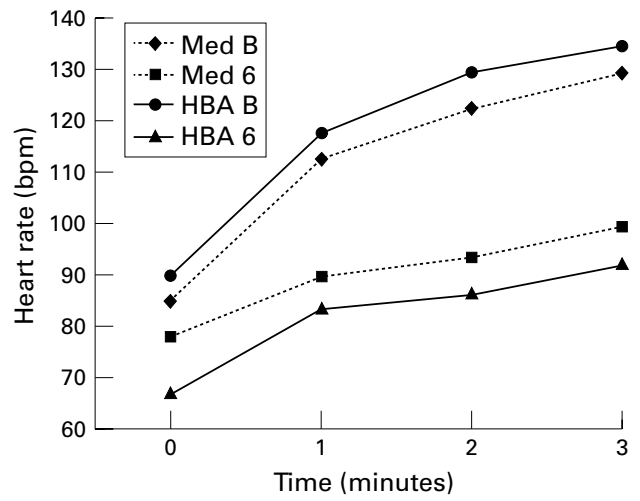

Figure 2 Example of heart rate profile on exercise in both groups for baseline (Med $B$, medical group baseline; $H B A$ $B$, ablation group baseline) and at six months follow up (Med 6, medical group at six months; HBA 6, ablation group at six months). Profile shown at one minute intervals for the first three minutes. Intra-group comparison (Med B $v$ Med 6 and $H B A B v H B A$ 6) showed a significant slowing in heart rate at all time intervals for both groups except the resting heart rate in the medical group at six months that had not significantly changed from baseline. Inter-group comparison at baseline (Med B v HBA B) showed no significant difference in heart rate profile for any of the time intervals, while at six months (Med $6 v$ HBA $6)$ the only significant difference was in the resting heart rate $(p=0.015)$. significantly slower in the HBA group throughout the study compared to the Med group. However, the heart rate profiles on exercise at one minute intervals did not differ.

MODIFIED KAROLINSKA QUESTIONNAIRE

In the Med group there was a significant improvement in the total $\mathrm{KQ}$ scores at all follow ups, with a mean reduction of approximately $50 \%$. For the individual symptoms there was significant improvement in chest pain, palpitations, dizziness, and shortness of breath at all follow up visits. By 12 months general health and activity level had also significantly improved, so that all individual symptoms had improved at this stage (table 3 ).

In the HBA group there was a significant improvement in the total $\mathrm{KQ}$ scores at all follow ups, again with a mean reduction of approximately $50 \%$. For the individual symptoms there was only significant improvement in palpitations and shortness of breath at all follow up visits. Symptoms of dizziness had improved by one month but this was not maintained. However, by 12 months general health and activity level had also significantly improved. Therefore four out of six specific symptoms had improved at this stage.

For inter-group comparison (Med $v$ HBA) there was no significant differences in any baseline results or between the groups at follow up (full statistical results not shown).

NOTTINGHAM HEALTH PROFILE

In the Med group for part 1 of the NHP there was a significant improvement in the total score at all follow ups. The mean reduction was approximately $40 \%$. For the individual dimensions physical mobility, pain, and emotional reaction were improved at all follow up visits and energy for the first six months (table 3).

In the HBA group for part 1 of the NHP there was again a significant improvement in the total score at all follow up visits. The mean reduction was less at approximately $20 \%$. For the individual dimensions only physical mobility had improved with benefit seen at all follow ups.

For inter-group comparison (Med $v$ HBA) for part 1 there was no significant differences in any baseline results or between the groups at follow up (full statistical results not shown).

For part 2 of the NHP there was no significant change from baseline in either group at any time or any difference between the groups 
Table 3 Results for modified Karolinska questionnaire (KQ) and the Nottingham health profile (NHP) for both groups (Med, medical group; HBA, ablation group) at baseline line and all follow ups

\begin{tabular}{|c|c|c|c|c|c|c|c|c|c|c|}
\hline & \multicolumn{2}{|l|}{ Baseline } & \multicolumn{2}{|l|}{1 month } & \multicolumn{2}{|l|}{3 months } & \multicolumn{2}{|l|}{6 months } & \multicolumn{2}{|l|}{12 months } \\
\hline & $\begin{array}{l}\text { Med } \\
(n=18)\end{array}$ & $\begin{array}{l}H B A \\
(n=18)\end{array}$ & $\begin{array}{l}\text { Med } \\
(n=18)\end{array}$ & $\begin{array}{l}H B A \\
(n=18)\end{array}$ & $\begin{array}{l}\text { Med } \\
(n=18)\end{array}$ & $\begin{array}{l}H B A \\
(n=18)\end{array}$ & $\begin{array}{l}\text { Med } \\
(n=16)\end{array}$ & $\begin{array}{l}H B A \\
(n=18)\end{array}$ & $\begin{array}{l}\text { Med } \\
(n=16)\end{array}$ & $\begin{array}{l}H B A \\
(n=16)\end{array}$ \\
\hline KQ total score $(0-140)$ & $50(22)$ & $48(26)$ & $18(13)^{\star}$ & $25(24)^{\star}$ & $26(18)^{\star}$ & $25(18)^{\star}$ & $26(18)^{\star}$ & $23(20)^{\star}$ & $22(17)^{\star}$ & $20(18)^{\star}$ \\
\hline Chest pain $(0-40)$ & $9.5(9.5)$ & $5.6(6.7)$ & $4.5(5.1)^{\star}$ & $4.1(5.3)$ & $6.1(7.3)^{\star}$ & $2.9(4.7)$ & $6.0(7.2)^{\star}$ & $3.5(4.9)$ & $4.7(5.0)^{\star}$ & $2.9(4.3)$ \\
\hline Palpitations $(0-30)$ & $13.3(7.7)$ & $13.6(9.0)$ & $2.4(3.2)^{\star}$ & $4.4(6.3)^{\star}$ & $3.8(3.3)^{\star}$ & $2.6(3.2)^{\star}$ & $4.1(4.1)^{\star}$ & $2.8(3.6)^{\star}$ & $4.5(4.6)^{\star}$ & $2.1(2.5)^{\star}$ \\
\hline Dizziness $(0-20)$ & $8.1(6.2)$ & $6.6(6.8)$ & $1.8(2.3)^{\star}$ & $3.4(5.8)^{\star}$ & $3.1(3.1)^{\star}$ & $4.2(5.2)$ & $4.3(3.8)^{\star}$ & $3.6(4.7)$ & $1.9(2.5)^{\star}$ & $4.0(4.9)$ \\
\hline Shortness of breath $(0-30)$ & $12.4(5.8)$ & $13.5(7.9)$ & $4.6(4.0)^{\star}$ & $6.3(6.6)^{\star}$ & $7.8(5.5)^{\star}$ & $8.9(6.9)^{\star}$ & $6.9(5.0)^{\star}$ & $7.0(7.2)^{\star}$ & $6.7(5.2)^{\star}$ & $6.0(5.2)^{\star}$ \\
\hline General health $(0-10)$ & $3.3(2.6)$ & $3.9(3.2)$ & $2.0(1.8)$ & $2.8(2.7)^{\star}$ & $2.5(2.0)$ & $2.6(1.8)$ & $2.1(2.0)$ & $2.5(2.2)$ & $1.4(1.2)^{\star}$ & $2.0(2.0)^{\star}$ \\
\hline Activity $(0-10)$ & $3.7(3.0)$ & $4.6(3.2)$ & $2.3(1.5)$ & $4.4(3.3)$ & $3.0(1.9)$ & $3.3(2.3)$ & $2.9(2.5)$ & $3.4(2.7)$ & $2.5(2.2)^{\star}$ & $3.2(2.9)^{\star}$ \\
\hline NHP total score $(0-600)$ & $120(90)$ & $145(99)$ & $61(80)^{\star}$ & $105(140)^{\star}$ & $57(64)^{\star}$ & $118(133)^{\star}$ & $60(65)^{\star}$ & $109(134)^{\star}$ & $52(63)^{\star}$ & $80(116)^{\star}$ \\
\hline Physical mobility $(0-100)$ & $22(17)$ & $29(21)$ & $8(14)^{\star}$ & $15(22)^{\star}$ & $7(12)^{\star}$ & $18(20)^{\star}$ & $8(13)^{\star}$ & $18(20)^{\star}$ & $9(17)^{\star}$ & $12(13)^{\star}$ \\
\hline Pain $(0-100)$ & $14.3(21)$ & $6.9(11)$ & $6.5(15)^{\star}$ & $5.8(19)$ & $4.8(9)^{\star}$ & $11(22)$ & $8.3(15)^{\star}$ & $5.9(11)$ & $5.0(13)^{\star}$ & $4.0(7.5)$ \\
\hline Sleep $(0-100)$ & $21(22)$ & $34(32)$ & $17(28)$ & $29(35)$ & $20(29)$ & $30(31)$ & $17(24)$ & $29(35)$ & $12(22)$ & $25(31)$ \\
\hline Energy $(0-100)$ & $39(38)$ & $44(41)$ & $17(28)^{\star}$ & $35(42)$ & $16(32)^{\star}$ & $34(41)$ & $18(25)^{\star}$ & $28(34)$ & $21(25)$ & $22(35)$ \\
\hline Social isolation $(0-100)$ & $4.8(10)$ & $9.4(15)$ & $4.8(16)$ & $5.6(23)$ & $2.3(7)$ & $10(25)$ & $3.7(11)$ & $10(25)$ & $3.0(10)$ & $7.0(26)$ \\
\hline Emotional reaction $(0-100)$ & $19(22)$ & $22(23)$ & $8.8(17)^{\star}$ & $15(27)$ & $6.9(13)^{\star}$ & $15(28)$ & $8.5(15)^{\star}$ & $19(30)$ & $3.1(7.3)^{\star}$ & $13(28)$ \\
\hline
\end{tabular}

A higher score means worse symptoms.

${ }^{\star} \mathrm{p}<0.05$ for intra-group comparison with baseline measurements. There was no significant difference in the results for inter-group comparison of Med $v$ HBA at all follow ups.

(full statistical results not shown). Figure 3 shows an example of results at six months.

\section{HOUR TAPE}

The mean (SD) heart rate over 24 hours at one month was significantly higher in the Med group compared to the HBA group (Med 83 (8) bpm $v$ HBA 71 (6) bpm, p < 0.01). These values and the difference between the two groups did not significantly change with further follow up.

The maximum (SD) heart rate from the 24 hour tape at one month did not significantly differ between the two groups (Med 116 (19) bpm $v$ HBA 113 (17) bpm, p = 0.6). These values also did not change significantly with further follow up.

The minimum heart rate was significantly different between the groups $(p<0.001)$. This was due to all patients pacing at their lower programmed rate (Med $70 \mathrm{bpm}, \mathrm{HBA}$ $60 \mathrm{bpm}$ ) at some time during the 24 hour tape, at all follow ups.

\section{ECHOCARDIOGRAM}

In both groups there was no significant change in any parameters compared to baseline at any follow up. For inter-group comparison there was again no significant differences at any stage.

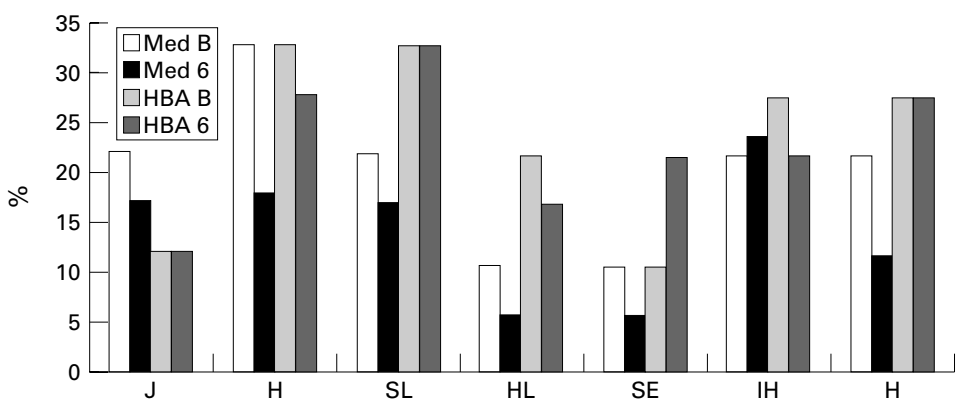

Figure 3 Example of part 2 NHP results at baseline and six months showing the percentage of patients answering yes to each question. There was no significant difference either intra-group or inter-group. Med B, medical group baseline; Med 6, medical group at six months; HBA B, ablation group baseline; HBA 6, ablation group at 6 months; $\mathcal{F}$, occupation; $H$, ability to perform jobs around the home; $S L$, social life; HL, home relationships; SE, sex life; $I H$, interest/hobbies; $H$, holidays.

\section{Discussion}

We have shown that, in patients with permanent $\mathrm{AF}$ with a mixed ventricular response rate, improved rate control will lead to a significant improvement in exercise duration and quality of life with equal preservation of a normal left ventricular function. However, rate regulation by ablation conferred no additional benefit compared to rate control alone in this patient group.

Patients with permanent AF often complain of limited exercise capacity and breathlessness despite a normal left ventricular function. In these patients assessment of ventricular rate control must be done thoroughly. Reliance on the ventricular rest rate at clinic visits is inadequate, as it gives no indication of the heart rate response to exercise. In this study the resting heart rate prior to exercise in the medical group did not significantly change from baseline at any follow up. However, at baseline there was poor rate control evident on exercise testing. Therefore assessment for rate control should include exercise testing to examine the heart rate profile and 24 hour Holter monitoring. Treatment to improve rate control will then lead to improved exercise capacity and quality of life.

In our study the exercise duration was increased by utilising either His bundle ablation plus VVIR pacemaker or by increasing the dosage of atrioventricular modifying drugs with a VVI pacemaker. Both treatments showed a significant and similar reduction in the heart rate response over the first three minutes of exercise compared to baseline. Despite this improved exercise capacity, neither strategy resulted in an improved $\mathrm{Vo}_{2} \mathrm{max}$, which is consistent with previous studies. ${ }^{3}$

Twenty four hour Holter monitor at follow up confirmed good overall heart rate control was achieved with either treatment, as the maximum heart rates were similar between the two groups. The mean heart rate was lower in the ablation group compared to the medical group, because of the lower base rate chosen (60 bpm $v 70 \mathrm{bpm}$ ).

Two tests-a cardiac specific test and a general health profile - were used to assess quality 
of life, which improved significantly with either treatment. However, there was a tendency for greater improvement in the medical group for individual subgroup symptoms. In part 2 of the general NHP there were no significant changes at any time, probably reflecting the age of our population (mean 69 years). The part 2 questions had a very low impact at baseline, so any change with treatment would be difficult to demonstrate.

The most frequently used drugs (15 patients, $83 \%$ ) in the medical group were the non-dihydropyridine calcium antagonists verapamil or diltiazem. They were chosen as previous studies have shown a neutral or positive benefit on exercise duration and an equivalent rate controlling effect to $\beta$ blockers, which tend to have a negative effect on exercise duration..$^{22-29}$ The majority of patients (13, $72 \%$ ) also required more than one drug. Our study further emphasised the poor rate controlling ability of digoxin, which is still frequently prescribed as a first line agent despite no study demonstrating its benefit. ${ }^{28}{ }^{29}$ Seventy five per cent of our patients took digoxin at baseline where rate control was inadequate. Digoxin does have a role as an add-on agent to other drugs. $^{30}$

His bundle ablation was expected to give additional benefit over rate control alone due to rate regulation. Uncontrolled prospective data had shown that rate regulation secondary to ablation improved ventricular function in heart failure patients even with so called controlled ventricular rates $(60-100 \mathrm{bpm})$ at baseline in the absence of any atrioventricular modifying drugs. ${ }^{1314}$ Furthermore, the deleterious effects of irregular right apical pacing compared to regular pacing with the same mean heart rate had been demonstrated in both experimental animal models and in humans. ${ }^{31-33}$ These studies showed irregular pacing caused a decrease in cardiac output with an increase in pulmonary capillary wedge pressure compared to regular pacing.

These studies, however, just demonstrated that irregular pacing is worse than regular pacing. They give no information on the comparison between an irregular rhythm with normal intrinsic conduction (baseline for AF patients) to a regular paced rhythm (following ablation) with the same heart rates. In our study, however, equivalent rate control assessed by exercise testing and 24 hour Holter monitoring was achieved with both treatments. The medical group had an irregular rhythm with normal conduction except when pacing at their lower base rate while the ablation group had a regular paced rhythm. Therefore, as both treatments had similar and significant positive benefits, this suggests rate control is more important than rate regulation in our patients with permanent $\mathrm{AF}$.

There is only one other randomised controlled study of His bundle ablation in patients with permanent AF. It compared ablation and VVIR pacemaker insertion with continued medical treatment with six months follow up in patients with moderately impaired left ventricular function (mean ejection fraction
$46 \%) .^{34}$ The benefits of ablation were not as great as previously estimated from uncontrolled studies. Quality of life was improved with both treatments, with ablation superior to drug treatment. However, neither treatment altered cardiac performance assessed with echocardiogram and exercise testing.

A further study in patients with permanent $\mathrm{AF}$ and moderately impaired left ventricular function (mean ejection fraction $40 \%$ ) investigated ablation and VVIR pacemaker insertion where a controlled ventricular rate was present at baseline because of atrioventricular modifying drugs. ${ }^{35}$ This non-randomised study consecutively consigned patients to one of three treatments. Firstly ablation, VVIR pacemaker implantation, and drug withdrawal (group 1), secondly, the same procedure with AV modifying drugs continued (group 2), and finally VVIR pacemaker implantation without ablation and continued medication (control group, group 3). The first two groups had a significant improvement in quality of life at six months, while there was no significant change in the third group. Left ventricular function assessed by echocardiography improved slightly in group 1 patients (from $39 \%$ to $45 \%$ ), with no change observed in the other two groups. Exercise duration was only assessed in group 1 patients, which showed no change from baseline.

This study therefore suggests that ablation producing rate regulation had a positive benefit on quality of life even when ventricular rate is adequately controlled at baseline with drugs. Furthermore the withdrawal of these drugs will lead to a small improvement in left ventricular function, while ablation alone will not. However, this study has several limitations. Firstly it was non-randomised. There was also no exercise data in the final two groups of patients and 24 hour tapes were not recorded in any patient. Therefore, it provides no evidence that rate control (or rate profile on exercise) was equivalent in all three groups or if there was any change from baseline. Therefore any benefit may be caused by alteration in rate control rather than rate regulation.

Overall these two studies together with our own cover a reasonable spectrum of left ventricular function. They suggest ablation may be advantageous when left ventricular function is moderately impaired irrespective of ventricular rate control. However, ablation offers no advantage in the presence of normal ventricular function if rate control can be achieved with drugs, with or without bradycardic support from a pacemaker. There are no controlled data currently available for patients with severely impaired left ventricular function (ejection fraction $<35 \%$ ) to guide treatment.

There are, however, patients with permanent $\mathrm{AF}$ that are drug refractory with a rapid ventricular response rate despite multiple drug combinations. These patients are different to our patients in this study, which were drug intolerant because of bradycardic side effects rather than being drug refractory. In these drug refractory patients' ablation and VVIR pacemaker is the ideal treatment. 
STUDY LIMITATIONS

We are limited by incomplete follow up at some of the later stages in this study. However, the majority of significant changes had occurred by three months with complete follow up available at this stage with changes generally remaining static thereafter.

We studied a specific population in permanent $\mathrm{AF}$ (represented $7 \%$ of total pacemakers implanted over the period of study recruitment), so our results can only be applied to them. However, we believe our data support the concept that ventricular rate control is important in any patient with $\mathrm{AF}$ and a normal ventricle.

Our study was not blinded as patients were aware that they underwent ablation and pacing spikes were observed during exercise testing at follow up, making it impossible to blind the research investigators. Furthermore different base rates were selected for the two groups, with a lower rate in the ablation group (60 $v$ 70). It is possible this may have affected our results as AF patients may benefit from higher rates. However, on exercise a similar increase in heart rate was achieved in both groups.

Some of the improved quality of life may be caused by bradycardic support rather than improved rate control as pacemaker implantation was also indicated for bradycardic episodes. Bradycardic support, however, would not explain our improved exercise capacity.

It is possible our study was undersized to demonstrate any difference between the two techniques. It was a similar size to previous studies evaluating ablation plus pacing or drug treatment in permanent AF. Furthermore, if any benefit could be demonstrated between the two techniques with larger numbers we feel it is less important than the benefit obtained from improved rate control achieved with either technique.

The negative inotropic effect of right ventricular pacing may also be important in explaining the results we have observed. Any benefit from rate regulation over and above rate control may be masked by this negative inotropic effect. Therefore the true benefit of rate regulation may be underestimated in this study. Newer pacing techniques such as biventricular pacing combined with ablation may give further advantages over right apical pacing or medical treatment and may be able to demonstrate that rate regulation is important.

\section{CONCLUSION}

For patients with normal left ventricular function in permanent $\mathrm{AF}$ with a mixed ventricular response rate, either His bundle ablation and VVIR pacemaker or atrioventricular modifying drugs with VVI pacemaker will lead to a significant improvement in exercise duration and quality of life with equal preservation of left ventricular function. Neither technique shows any significant advantage over the other, with equal improvement in rate control. This suggests the positive benefits of ablation and pacing are caused by improved rate control rather than rate regulation in patients with a normal ventricle. However, we would recom- mend medical treatment first with a VVIR pacemaker (programmed VVI) as it avoids initial irreversible ablation. Ablation can be performed at a later stage in non-responders.

1 Brignole M, Gianfranchi L, Menozzi C, et al. Influence of the atrioventricular junction radiofrequency ablation in patients with chronic atrial fibrillation and flutter on quality of life and cardiac performance. $A m \quad \mathcal{F}$ Cardiol 1994;74:242-6.

2 Twidale N, Sutton K, Bartlett L, et al. Effects on cardiac performance of atrioventricular node catheter ablation using radiofrequency current for drug refractory atrial arrhythmias. PACE 1993;16:1275-84.

3 Buys EM, van Hemel NM, Kelder JC, et al. Exercise capacity after His bundle ablation and rate response ventricular pacing for drug refractory chronic atrial fibrillation. Heart 1997;77:238-41.

4 Edner M, Caidahl K, Bergfeldt L, et al. Prospective study of left ventricular function after radiofrequency ablation of atrioventricular junction in patients with atrial fibrillation. atrioventricular junction in

5 Rodriguez LM, Smeets JLRM, Xie B, et al. Improvement in Rodriguez LM, Smeets JLRM, Xie B, et al. Improvement in left ventricular function by ablation of atrioventricular tion. Am f Cardiol 1993;72:1137-41.

6 Rosenqvist M, Lee MA, Moulinier L, et al. Long-term follow up of patients after transcatheter direct current ablation of the atrioventricular junction. $\mathcal{F} \mathrm{Am}$ Coll Cardiol 1990;16;1467-74.

7 Fitzpatrick AP, Kourouyan HD, Siu A, et al. Quality of life and outcomes after radiofrequency His-bundle catheter ablation and permanent pacemaker implantation: Impact of treatment in paroxysmal and established atrial fibrillation. Am Heart f 1996;131:499-507.

8 Jenkins LS, Ellenbogen KA, Kay GN, et al. Quality of life post ablation/pacemaker implantation in patients with symptomatic atrial fibrillation. Circulation 1996;94(suppl): 3399 .

9 Ellenbogen KA, Kay GN, Giudici MG, et al. Radiofrequency ablation of the AV junction improves functional quency ablation of the AV junction improves functional the APT trial. Circulation 1996;94(suppl):3984.

10 Heinz G, Siostrzonek P, Kreiner G, et al. Improvements in left ventricular systolic function after successful radiofrequency His bundle ablation for drug refractory, chronic atrial fibrillation and recurrent atrial flutter. Am 7 Cardiol 1992;69;489-92.

11 Clark DM, Plumb VJ, Kay GN. The haemodynamics of atrial fibrillation: the independent effect of an irregular RR interval. Circulation 1995;92 (suppl):666.

12 Daoud E, Weiss R, Bahu M, et al. Effect of a regular and irregular ventricular rhythm on cardiac output. PACE 1996;19(suppl):502.

13 Natale A, Zimmerman L, Tomassoni G, et al. Impact on ventricular function and quality of life of transcatheter ablation of the atrioventricular junction in chronic atrial fibrillation with a normal ventricular response. Am $f$ Cardiol 1996;78:1431-3.

14 Helguera ME, Pinski SL, Khoudeir Y, et al. Improvement in left ventricular systolic function after successful radiofrequency $\mathrm{AV}$ junctional ablation in patients with chronic quency AV junctional ablation in patients with chronic 1994;17(suppl):233

15 Le Tourneau T, Klug D, Lacroix D. Mitral valve replacement for pacing-induced severe mitral regurgitation after radiofrequency ablation of the atrioventricular node. Heart 1996;76:457.

16 Vanderheyden M, Goethalis M, Anguera I, et al. Haemodynamic deterioration following radiofrequency ablation of the atrioventricular conduction system. PACE 1997;20: 2422-8.

17 Cannan C, Higano S, Holmes D. Pacemaker induced mitral regurgitation: an alternative form of pacemaker syndrome. PACE 1997;20:735-8.

18 Tse H, Lau C. Long-term effect of right ventricular pacing on myocardial perfusion and function. $\mathcal{f} \mathrm{Am}$ Coll Cardiol 1997;29:744-9.

19 Linde C. How to evaluate quality of life in pacemaker patients: problems and pitfalls. PACE 1996;19:391-7.

20 Pocock S, Henderson R, Seed P, et al. Quality of life, employment status, and anginal symptoms after coronary angioplasty or bypass surgery. 3-year follow-up in the randomized intervention treatment of angina (RITA) trial. Circulation 1996;94:135-42.

21 Caine N, Harrison S, Sharples L, et al. Prospective study of quality of life before and after coronary artery bypass grafting. BMF 1991;302:511-16.

22 Lewis RV, McMurray J, McDevitt DG. Effects of atenolol, verapamil, and xamoterol on heart rate and exercise tolerance in digitalised patients with chronic atrial fibrillation. $\mathcal{F}$ Cardiovasc Pharmacol 1989;13:1-6.

23 James MA, Channer KS, Papouchado M, et al. Improved control of atrial fibrillation with combined pindolol and digoxin therapy. Eur Heart $\mathcal{7}$ 1989;10:83-90.

24 Singh S, Saini RK, DiMarco J, et al. Efficacy and safety of sotalol in digitalised patients with chronic atrial fibrillation. Am $\mathcal{F}$ Cardiol 1991;68:1227-30.

25 Atwood JE, Sullivan M, Forbes S, et al. Effect of beta-adrenergic blockade on exercise performance in patients with chronic atrial fibrillation. F Am Coll Cardiol 1987;10:314-20. 
26 Lundstrom T, Ryden L. Ventricular rate control and exercise performance in chronic atrial fibrillation: effects of diltiazem and verapamil. f Am Coll Cardiol 1990;16:86-90.

27 Lewis RV, Irvine N, McDevitt DG. Relationship between heart rate, exercise tolerance and cardiac output in atrial fibrillation: the effects of treatment with digoxin, verapamil and diltiazem. Eur Heart f 1988;9:777-81.

28 Wong CK, Lau CP, Leung WH, et al. Usefulness of labetalol in chronic atrial fibrillation. Am f Cardiol 1990;66:1212-15.

29 Matsuda M, Matsuda Y, Yamagishi T, et al. Effects of digoxin, propranolol, and verapamil on exercise in patients with chronic isolated atrial fibrillation. Cardiovasc Res 1991;25:453-7.

30 Lewis RV, Laing E, Moreland TA, et al. Comparison of digoxin, diltiazem and their combination in the treatment of atrial fibrillation. Eur Heart f 1988;9:279-83.

31 Naito M, David D, Michelson E, et al. The hemodynamic consequences of cardiac arrhythmias: evaluation of the relative roles of abnormal atrioventricular sequencing, irregularity of ventricular rhythm and atrial fibrillation in a canine model. Am Heart f 1983;106:284-91.

32 Daoud E, Weiss R, Bahu M, et al. Effect of an irregular ventricular rhythm on cardiac output. Am f Cardiol 1996;78: 1433-6.

33 Clark D, Plumb V, Epstein A, et al. Hemodynamic effects of an irregular sequence of ventricular cycle lengths during atrial fibrillation. f Am Coll Cardiol 1997;30:1039-45.

34 Brignole M, Menozzi C, Gianfranchi L, et al. Assessment of atrioventricular junction ablation and VVIR pacemaker versus pharmacological treatment in patients with heart failure and chronic atrial fibrillation. A randomised, controlled study. Circulation 1998;98:953-60.

35 Natale A, Zimerman L, Tomassoni G, et al. AV node ablation and pacemaker implantation after withdrawal of effective rate-control medications for chronic atrial fibrillation: effect on quality of life and exercise performance. PACE 1999;22:1634-9.

\section{Thrombus overlying the main stem crista: a three dimensional reconstruction}

A 67 year old patient was referred to our hospital for diagnostic catheterisation. For six days she had been suffering from unstable angina, which was stabilised medically. She had no coronary risk factors. The diagnostic angiogram showed a large thrombus reaching from the first diagonal branch through the proximal left anterior descending artery (LAD), overlying the main stem crista and reaching distally of the first marginal branch into the left circumflex artery (LCX). Intravascular ultrasound (IVUS) of the LAD showed a smooth, organised thrombus crossing the first diagonal and septal branch of the LAD without presence of atherosclerotic disease of the artery. The three dimensional reconstruction shows the thrombus alongside a normal arterial wall. The patient was treated with abciximab bolus and 24 hour infusion. She remained free of symptoms and was again catheterised four days later. Angiography and IVUS showed complete dissolution of the thrombus, and the patient was discharged from the hospital. As the control angiogram and IVUS images revealed no significant coronary artery disease, we hypothesised a left cardiac or paradoxical (in the case of an atrial or ventricular septal defect or a patent foramen) origin of the thrombus. Transthoracic and oesophageal echocardiograms, however, revealed no thromboembolic origin. Possibly, rupture of a minimal plaque marked the onset of the acute coronary syndrome. On aspirin treatment, she remains free of cardiac complaints four months after the initial event.

GLENN VAN LANGENHOVE PAUL VERMEERSCH PATRICK W SERRUYS

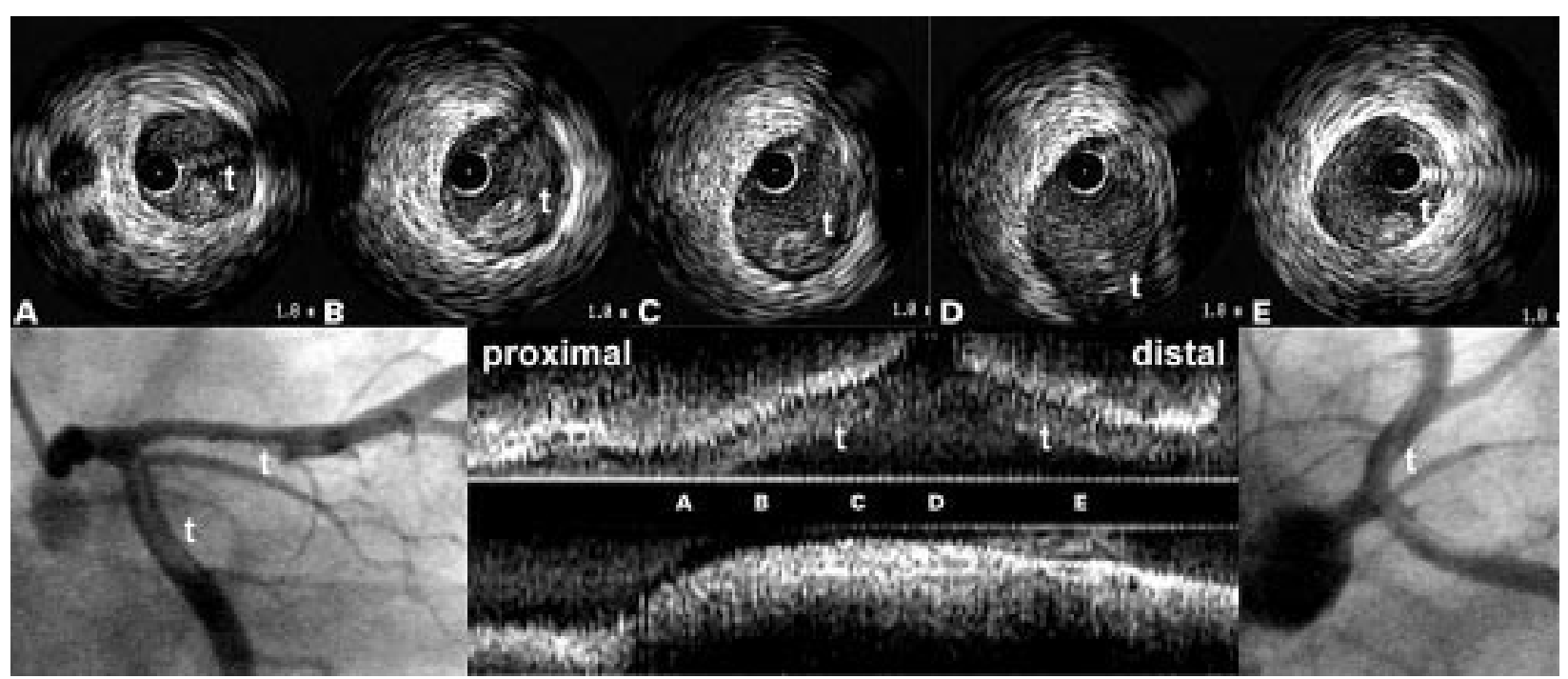

Coronary angiogram (RAO view) of LAD (top) and CX (bottom) in the lower left corner, showing thrombus ( $t$ ) overlying the main stem crista. The lower right picture shows the LAO view. The lower centre shows an IVUS longitudinal reconstruction of the LAD with the thrombus ( $t)$ along the wall of the $L A D$. The upper pictures $(A-E)$ show the respective IVUS cross sections corresponding with $A-E$ in the longitudinal reconstruction. Picture $D$ shows thrombus crossing the first septal branch. 